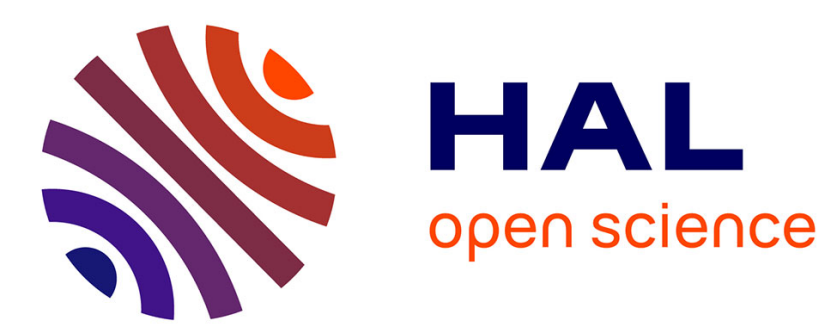

\title{
Diagnosing mechanical changes in vibrating systems
}

George V. Moustakides, Michèle Basseville, Albert Benveniste, Georges Le Vey

\section{To cite this version:}

George V. Moustakides, Michèle Basseville, Albert Benveniste, Georges Le Vey. Diagnosing mechanical changes in vibrating systems. [Research Report] RR-0942, INRIA. 1988. inria-00075616

\section{HAL Id: inria-00075616 https://hal.inria.fr/inria-00075616}

Submitted on 24 May 2006

HAL is a multi-disciplinary open access archive for the deposit and dissemination of scientific research documents, whether they are published or not. The documents may come from teaching and research institutions in France or abroad, or from public or private research centers.
L'archive ouverte pluridisciplinaire HAL, est destinée au dépôt et à la diffusion de documents scientifiques de niveau recherche, publiés ou non, émanant des établissements d'enseignement et de recherche français ou étrangers, des laboratoires publics ou privés. 

n.t. in bo xin th

$3 x$

4.

\% W

3.t. 3.x.

H.

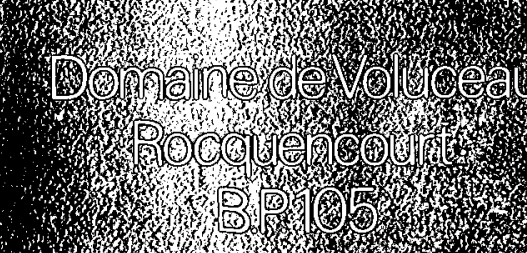

1030 3

Wx.

(3)

หัง

\section{Rapports de Recherche}

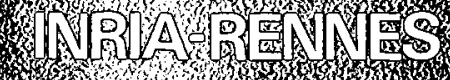
20.

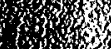

(3)

3.

\%

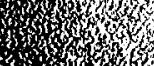

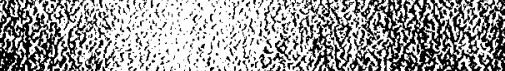

is

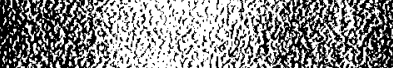

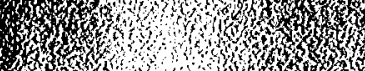

H

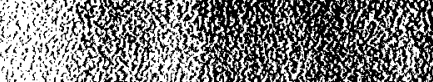

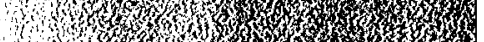
ำ

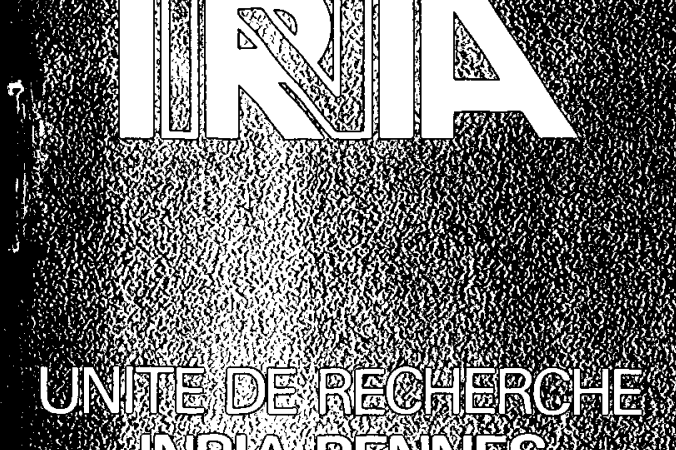
放, to wow x. ,

,

\section{CHANGES IN VIBRATING SYSTEMS \\ DIAGNOSING MECHANICAL

\section{CHANGES IN VIBRATING SYSTEMS}

No 942

\section{Programme 5}

George MOUSTAKIDES Michèle BASSEVILLE Albert BENVENISTE Georges LE VEY

Décembre 1988 
DIAGNOSING MECHANICAL CHANGES IN VIBRATING SYSTEMS

George MOUSTAKIDES, Michèle BASSEVILLE Albert BENVENISTE, Georges LE VEY

Publication Interne $\mathrm{n}^{\circ} 436$

Octobre 1988

Yr

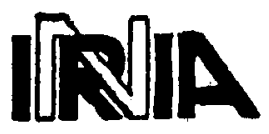

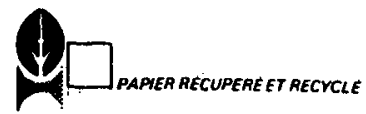


Campus Universitaire de Beaulieu

35042-RENNES CÉDEX

FRANCE

Téléphone: 99362000

Télex: UNIRISA $950473 \mathrm{~F}$

Télécopie: 99383832

\title{
Diagnosing mechanical changes in vibrating systems
}

\section{George Moustakides +,Michèle Basseville *,Albert Benveniste **, Georges Le Vey ++}

\author{
Publication Interne $n^{\circ} 436$ - Octobre 1988 - 12 Pages
}

\begin{abstract}
We describe the key steps and algorithms of a general approach for fault diagnosis we have recently proposed, for the particular case of diagnosis of changes in a vibrating system in terms of the mechanical model. Our method is based upon a validation criterion between new measurements and a nominal small identifiable black-box model. The diagnosis in terms of the complex non identifiable physical model of the system is obtained via sensitivity tests along synthetical jacobians which achieve the necessary reduction between the two types of models in a convenient way.
\end{abstract}

\section{Diagnostic physique en surveillance des vibrations}

RÉSUMÉ: Nous décrivons les principales étapes et les algorithmes d'une approche générale de diagnostic que nous avons récemment proposée, dans le cas particulier du diagnostic de changements de caractéristiques vibratoires en termes du modèle mécanique. Notre méthode est basée sur un critère de validation entre de nouvelles mesures et un modele nominal de type "boite noire" petit et identifiable. Le diagnostic en termes du modele physique complexe et non identifiable est obtenu à l'aide de tests de sensibilité le long de jacobiens synthétiques qui réalisent, de manière convenable, la réduction nécessaire entre les deux types de modèles utilisés.

This work was supported by IFREMER grant no 86.2.330516.DIT.GO.S.

+ Presently at C.T.I., P.O.Box 1122, Patras 261 10, Greece.

++ Presently at I.N.R.I.A. Rocquencourt.

* Also with C.N.R.S.

** Also with I.N.R.I.A. 


\section{Introduction}

The problem of change or fault detection and diagnosis in dynamic systems has received a growing attention, reflecting the importance of this key issue in many industrial areas. A substantial amount of static or dynamic model-based techniques have been developed, as can be seen from the survey papers: Willsky,1976; Isermann,1984; Frank, 1987; Basseville,1988. Two types of diagnosis situations are basically to be distinguished (Benveniste et al.,1987):

1. diagnosis of changes on identifiable model sets: in this case, there is a one-to-one mapping between the parameters used for detecting model changes and the parameters for which diagnosis is desired. For example, you monitor an AR or ARMA model and wish to know which poles actually moved. Many model-based fault diagnosis techniques explicitly assume to deal with this restricted situation (Isermann,1984). Another typical example is given by the detection filters approach (White and Speyer, 1987) where special types of observers are designed for failure detection and diagnosis.

2. diagnosis of changes on non-identifiable model sets: this situation is much more difficult to solve because the convenient parameterization for diagnosis is in terms of non identifiable parameters. For example, you wish to monitor (and to diagnose changes in) a complex system modelled by a large non-identifiable physical model.

We address here the second type of diagnosis problem in the particular situation of vibration monitoring, for which we already presented new statistical tools for change detection and (modal) diagnosis of type 1 (Basseville et al., 1987). Our solution for diagnosis on non identifiable model sets is more general as discussed in (Benveniste et al.,1987). We basically use a small identifiable blackbox model as the nominal model for monitoring, and hypotheses of change in the space of the non identifiable physical parameters. We describe here the different steps of the method in the particular case of mechanical systems for which a (not necessarily accurate) finite element model is assumed to be at hand.

The paper is organized as follows. In section 2 , we briefly recall our instrumental test for change detection and sensitivity method for diagnosis on identifiable model sets. In section 3 , we extend the sensitivity method for diagnosis on non identifiable model sets, and describe the computation of the synthetical jacobians, which achieve the model reduction between the physical model and the blackbox one in a way which is consistent with our statistical decision tool. Section 4 is devoted to the presentation of some numerical results on a simulated example, which outlines the ability of correctly diagnosing physically small changes. Conclusions are drawn in section 5. 


\section{Instrumental and sensitivity tests}

We have shown (Basseville et al.,1987) that a possible approach to vibration monitoring consists in detecting changes in the AR part of a multivariable ARMA process whose MA part is nonstationary and considered as a nuisance. The AR part contains all the vibrating characteristics which are accessible from the measurements, namely the frequencies and the observed part of the modal shapes, and is used as the black-box model referred above. Actually, we have solved the following model validation problem: given a nominal model (i.e. the signature of the safe vibrating system) in terms of $(r, r)$ AR matrix coefficients (where $r$ is the number of sensors): ${ }^{1}$

$$
\left(A_{p}^{o} \quad \ldots A P\right):=\theta_{o}^{T}
$$

and a new record of measurements, decide whether the signature still adequately represents the vibrating characteristics of the structure as measured in this record. For this purpose, we built a statistic:

$$
\chi\left(\theta_{o}, \delta \theta \in \theta,\left(Y_{\imath}\right)_{1 \leq t \leq s}\right)
$$

depending upon the nominal model $\theta_{o}$, an assumed change direction $\Theta$ and the new record. Using this statistics, the decision rule is as follows:

$$
\begin{gathered}
\chi<\text { threshold } \Rightarrow \delta \theta=0 \\
\chi \geq \text { threshold } \Rightarrow 0 \neq \delta \theta \in \theta
\end{gathered}
$$

For a global alarm, $\boldsymbol{\theta}$ is taken to be the set of all the possible $\theta$ 's. For focusing the monitoring on one mode or set of modes, we used a sensitivity method as follows. Let $\psi$ be another parameterization of the AR part $\theta$, for example the modal parameters, e.g. eigenvalues and eigenvectors of the polynomial matrix $\Sigma A_{i} z^{-i}$. Set:

$$
\theta=F(\Psi)
$$

Let $\Psi$ be the space spaned by a subset of coordinates of $\Psi$. We then choose:

$$
\boldsymbol{\theta}=\boldsymbol{F}^{\prime}\left(\Psi_{0}\right) . \Psi
$$

where $F^{\prime}\left(\psi_{o}\right)$ is the jacobian computed at the nominal model parameterized in $\psi$. We shall see in the next section how to extend this simple idea to the more complex case of diagnosis of type 2 . We now recall the definition of the statistics $\chi$ and the decision rule. Let

$$
U \mathcal{T}_{N}(s):=\left(\begin{array}{llll}
A_{p}^{o} & \ldots & A & -I) \mathrm{H}_{p+1, N}(s)
\end{array}\right.
$$

1 := means "equal by definition" 
be the instrumental statistics, where $H_{p+1, N}(s)$ is the Hankel matrix computed on the new record ${ }^{2}$. We have shown (Basseville et al., 1987)that the detection and diagnosis decision rules are of the following chi- squared type in the scalar case:

$$
\chi=U \hbar_{N}(s) \Sigma_{N}^{-1}(s) M\left(M^{T} \Sigma_{N}^{-1}(s) M\right)^{-1} M^{T} \Sigma_{N}^{-1}(s) U_{N}(s)
$$

where $\Sigma_{N}(s)$ is an estimate of the covariance matrix of $U$, and where $M$ is given by

$$
\begin{gathered}
\mathrm{H}_{p, N}^{T}(s) \text { for the global alarm } \\
\mathrm{H}_{p, N}^{T}(s) \mathrm{J}_{p, m} \text { for the modal diagnosis }
\end{gathered}
$$

where

$$
\mathrm{J}_{p, m}=\left(\frac{\partial A_{i}}{\partial \Psi_{j}}\right)_{i, j}
$$

In the multiple sensor case, the formulas can be easily extended (Basseville et al.,1987). Note that, for $N=p$ :

$$
\chi=U_{p}^{T}(s) \Sigma_{p}^{-1}(s) U_{p}(s)
$$

We actually use this test in practice.

\section{Physical diagnosis}

We now describe in detail how to extend the above diagnosis procedure - instrumental test (2-6) and sensitivity calculus (2-4) - to the case of physical diagnosis where a model reduction has to be achieved: the physical system (parameterized by the mass, damping and stiffness matrices - see (3-2)) may have several hundreds degrees of freedom, while the dimension of the parameter $\theta_{o}$ is usually less then 100 . In this case, we choose -instead of (2-4)- :

$$
\theta=F^{\prime}(\Psi *) . \Psi
$$

We assume here that an approximate physical model $\psi *$ of the structure is at hand. In (3-1), F' $(\psi *)$ is the jacobian at this nominal model. The section is organized as follows. We first show how to use an a priori knowledge about the physical system for computing a reduced model $\theta *$ and all the possible change directions corresponding to all the admissible changes in the physical parameters. We then show how to cluster these change vectors -which are generally too numerous- in order to obtain the directions of change which are then incorporated in (2-7) for the sensitivity tests (2-6). The change

2 see Basseville et al. 1987, formula (2-7) and subsequent for the undefined notations 
direction which produces the highest sensitivity test is considered to be the most likely origin of the change detected by the global test (2-8).

\subsection{Physical model and reduced model}

We use (Basseville et al.,1987) the following physical model:

$$
\left\{\begin{array}{c}
M \ddot{Z}+C \dot{Z}+K Z=E \\
Y=L Z
\end{array}\right.
$$

where $M, C, K$ are the mass, damping and stiffness matrices, given by the designer, and assumed to be symmetrical; $Z$ is the vector of displacement of the $m$ degrees of freedom; $E$ is a non-stationary white noise; and $Y$ is the $r$-dimensional measurement. In order to use our sensitivity approach, we need a jacobian (3-1) and thus a relation between the model $M, C, K$ and an associated (and not estimated) AR model of reduced size, since it is not possible to monitor all the modes of the system (3-2). We compute this model as follows. Let:

$$
\psi_{*}=\left(\lambda_{i}, \phi_{i}\right)_{1 \leq i \leq 2 m}
$$

be a selection of modes, pairwise conjugated. They satisfy:

$$
\left(\lambda_{i}^{2} M+\lambda^{i} C+K\right) \phi_{i}=0
$$

We shall call reduced AR model and denote by (Moustakides, 1986)

$$
\text { oT }:=\left(A_{p}^{*} \ldots A_{1}^{*}\right)
$$

the solution of the following system:

$$
B_{p}^{T}=B^{T} \theta
$$

where: $B^{T}=\left(\begin{array}{llll}B & 6 & \ldots & B_{p-1}^{T}\end{array}\right)$,

$$
B_{k}=L(\phi \bar{\phi}) e^{k \tau D}:=L T e^{k \tau D}
$$

In this formula, $\tau$ is the sampling frequency, $\phi$ contains the $m$ selected eigenvectors,

$$
D=\left(\begin{array}{ll}
\Delta & 0 \\
0 & \Delta
\end{array}\right)
$$

where $\Delta=\operatorname{diag}\left(\lambda_{i}\right)$ contains the corresponding eigenvalues. It is clear that $\theta *$ is not identical to $\theta_{o}$. 
To reduce the dissimilarity, for computing $\theta *$, we select, among the solutions of (3-3), those which are closest to the identified modes, and solve (3-5). Let us insist upon the fact that, in our minds this discrepancy between $\theta_{*}$ and $\theta_{o}$ is not crucial: we are interested in the directions of changes of the model and we hope that these directions do not alter significantly with respect to the nominal model where they are computed.

\subsection{Computation of the change vectors}

Assuming that we monitor only changes in the mass $M$ and stiffness coefficients $K$, we have to compute, for each $\delta M$ and $\delta K$, the corresponding change $\delta \theta$ in the nominal model $\theta *$. It can be easily shown (Moustakides, 1986) that the knowiedge of $\delta \lambda$ and $\delta \phi$ is sufficient for this purpose: simply differentiate (3-5). For getting these quantities, we use (Moustakides, 1986), a similar method as in (Stewart, 1973). Differentiating (3-3), we get:

$$
\delta \lambda(2 \lambda M+C) \phi+\left(\lambda^{2} M+\lambda C+K\right) \delta \phi+\left(\lambda^{2} \delta M+\delta K\right) \phi=0
$$

On the other hand, because of the symmetry of the matrices, we also have:

$$
\phi^{T}\left(\lambda^{2} M+\lambda C+K\right)=0
$$

Multiplying (3-6) from the left by $\phi^{T}$ and using (3-7) gives:

$$
\delta \lambda=-\frac{\phi^{T}\left(\lambda^{2} \delta M+\delta K\right) \phi}{\phi^{T}(2 \lambda M+C) \phi}
$$

Merging (3-8) and (3-6) yields

$$
\left(\lambda^{2} M+\lambda C+K\right) \delta \phi=-\delta \lambda(2 \lambda M+C) \phi-\left(\lambda^{2} \delta M+\delta K\right) \phi
$$

We look for the solution in $\delta \phi$ which is orthogonal to $\phi$, i.e.:

$$
\phi^{T} \delta \phi=0
$$

In practice, the damping matrix $C$ is not precisely known. As we are dealing with systems having low damping, we compute the change vectors in the case where $C=0$.

We conclude this subsection by noting that all the above computed change vectors cannot be reasonably discriminated. Actually, for a system with 144 degrees of freedom, there are about 20000 elementary changes $\delta M$ and $\delta K$; if the nominal model $\theta *$ is made of 20 modes and the number of sensors is 3 , the change vectors are in a space with dimension 60 . We thus cluster the resulting set of points, and the corresponding centers of gravity will be used as synthetical jacobians. 


\subsection{The metric used for clustering}

We naturally use the very metric associated to the chi-squared test (2-6). Similarly, we define a distance between two change vectors by the corresponding scalar product:

$$
d(i, j)=\frac{\left[\left(H_{p, p} I_{r}\right) \Theta_{i}\right]^{T} \Sigma_{p}^{-1}\left[\left(H_{p, p} I_{r}\right) \Theta_{j}\right]}{\left\|\Theta_{i}\right\|\left\|\Theta_{j}\right\|}
$$

In order to improve the quality of the clustering, it is necessary to reject the change vectors which are too small with respect to this metric.

\subsection{Clustering the resulting change vectors}

Because of the above metric (3-11), we are working with data on the unit sphere. Therefore, we have to use a classification method which does not destroy this geometry, and thus does not center and reduce the data. Because we do not know, a priori the number of classes, we use a hierarchical classification method.

\section{Numerical results}

We use the same simulated system as in (Basseville et al.,1987) which was motivated by the application to offshore platforms; see the figures below. Using measurements at nodes $7,13,12$, we show here that we are able to diagnose correctly a change $\mathrm{H} 1$ in the stiffness of the connection to the ground and a cutting $\mathrm{H} 2$ of the spring between the nodes 8 and 11 . We already know that we are able to detect these two types of changes, even though the second one is very small from a mechanical point of view. Using the above procedure, we generated 14 synthetical jacobians, along which sensitivity tests can be run for two sets of data simulating the two types of changes. The numerical results are presented in table 1 . The decision strategy consists in selecting the sensitivity test with maximal value, and diagnosing the change in terms of the mechanical elements which form the class underlying the corresponding synthetical jacobian. These classes are drawn in boldface on figures no 1 and 2 and show a quite satisfactory physical coherence, leading to a correct diagnosis.

\section{Conclusions}

We have presented the key steps of a new approach for diagnosing changes in non- identifiable modelsets, for the particular case of vibration monitoring. The preliminary results obtained on a simulated system have been shown to be quite encouraging, for diagnosing physically different changes as well 
as from the point of view of robustness with respect to the nonstationarity of the environment, which is a key issue in model-based fault diagnosis techniques (Frank, 1987). However, our approach is still too much naive from the mechanical point of view -we generate all the elementary changes in $M$ and $K$ without a priori mechanical information, and then cluster them- and cannot be applied straightforwardly on large systems. Further investigations in this direction are currently made for the case of turbo-machines.

\section{References}

- Basseville, M., A.Benveniste, G.Moustakides and A.Rougee (1987). Detection and diagnosis of changes in the eigenstructure of nonstationary multivariable systems. Automatica, 23, 479-489.

- Basseville, M. (1988). Detecting changes in signals and systems - A survey. Automatica; 24, 309-326.

- Benveniste, A., M.Basseville and G.Moustakides (1987). The asymptotic local approach to change detection and model validation. I.E.E.E. Trans. Aut. Control, 32, 583-592.

- Frank, P.M. (1987). Fault diagnosis in dynamic system via state estimation - A survey. In Tzafestas et al.(eds), Reliability and related knowledge-based approaches, vol.1, 35-98, D.Reidel Publishing company.

- Isermann, R. (1984). Process fault detection based on modeling and estimation methods - A survey. Automatica, 20, 387-404.

- Moustakides, G. (1986). The problem of diagnosis with respect to physical parameters for changes in structures. IRISA Research Report no 295.

- Stewart, H. (1973). Introduction to matrix computation. Academic Press, 289-306.

- White, J.E. and J.Speyer (1987). Detection filter design: spectral theory and algorithms. I.E.E.E. Trans. Aut. Control, 32, 593-603.

- Willsky, A.S. (1976). A survey of design methods for failure detection in dynamic systems. Automatica, 12, 601-611. 


$\begin{array}{ccc}\text { changetype } & H 1 & H 2 \\ \text { global test } & 1514.53 & 1623.37 \\ \text { sensitiv.1 } & 3.79 & 157.34 \\ \text { sensitiv.2 } & 193.42 & 18.35 \\ \text { sensitiv.3 } & 254.66 & 3.10 \\ \text { sensitiv.4 } & 58.13 & 195.52 \\ \text { sensitiv.5 } & 2.44 & 2.16 \\ \text { sensitiv.6 } & 0.14 & 1122.13 \\ \text { sensitiv.7 } & 0.11 & 1510.76 \\ \text { sensitiv.8 } & 410.60 & 0.06 \\ \text { sensitiv.9 } & 104.69 & 232.43 \\ \text { sensitiv.10 } & 0.01 & 120.69 \\ \text { sensitiv.11 } & 1414.67 & 2.28 \\ \text { sensitiv.12 } & 1.87 & 1362.60 \\ \text { sensitiv.13 } & 0.86 & 738.66 \\ \text { sensitiv.14 } & 1.01 & 748.42\end{array}$

\section{Table no 1:numerical values of the tests}
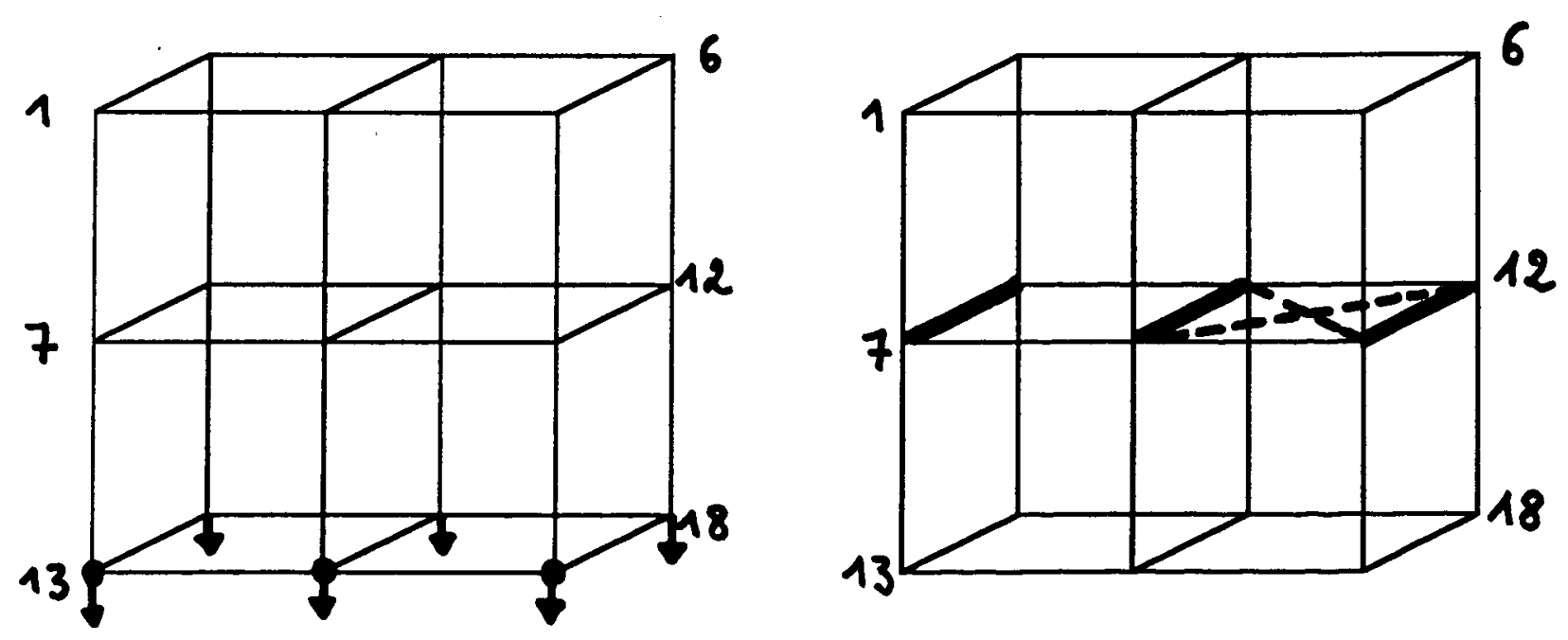

Figure no 1:content of class no 11 | Figure no 2:content of class no 7 
10

LIST'E DES DERNIERES PUBLICATIONS INTERNES

PI 432 - PYRAMIDE, LOGICIEL DE MODELISATION GEOMETRIQUE POUR LA TELEOPERATION. MANUEL UTILISATEUR ET GUIDE DU PROGRAMMEUR Philippe EVEN

94 Pages, Octobre 1988.

PI 433 - SPEAKER INDEPENDENT ACOUSTIC-PHONETIC RECOGNITION USING ADAPTATIVE VECTOR QUANTIZATION

Huan-Yu SU

16 Pages, Octobre 1988.

PI 434 - SIMULATING STRUCTURED PLANS FOR TELEROBOTICS USING TEMPORAL LOGICS

Eric RUTTEN, Lionel MARCE

16 Pages, Octobre 1988.

PI 435 - PERFORMANCE MODELLING OF QUEUES WITH RENDEZ VOUS SERVICE Louis-Marie LE NY, C. Murray WOODSIDE

24 Pages, Octobre 1988.

PI 436 - DIAGNOSING MECHANICAL CHANGES IN VIBRATING SYSTEMS George MOUSTAKIDES, Michèle BASSEVILLE, Albert BENVENISTE, Georges LE VEY

12 Pages, Octobre 1988. 


$$
\text { ' }
$$

